\title{
Técnica Anatómica para Restaurar y/o Reproducir Piezas Óseas Humanas de Difícil Obtención, para la Investigación y Docencia Científica
}

\author{
Anatomical Technique to Restore and/or Reproduce Human Bone \\ Pieces Difficult to Obtain for Research and Teaching Science
}

*Mauricio Villarroel Guerra \& **Eugenio Medina Otazo

VILLARROEL, G. M. \& MEDINA, O. E. Técnica anatómica para restaurar y/o reproducir piezas óseas humanas de difícil obtención, para la investigación y docencia científica. Int. J. Morphol., 29(2):532-536, 2011.

RESUMEN: Se describe una técnica para la reproducción y/o restauración de piezas óseas humanas con fines didácticos y académicos para uso científico y/o artístico. Esta técnica permite obtener preparaciones de gran calidad en textura, color y diversos parámetros morfológicos permitiendo obtener detalles y tridimensionalidad de las piezas óseas. La técnica está aplicada a la reproducción de piezas óseas humanas de difícil obtención, como son los huesos de la cabeza, sin embargo se pueden reproducir todo tipo de piezas óseas incluso con menor grado de dificultad. Nuestros resultados confirman que está técnica permite obtener un altísimo grado de fiabilidad comparado con la pieza original, por ello puede ser de gran utilidad en docencia e investigación.

PALABRAS CLAVE: Técnica anatómica; Acrílicos; Reproducciones óseas; Material didáctico.

\section{INTRODUCCIÓN}

Dentro del estudio práctico de la osteología humana ya sea artístico o científico, existen piezas óseas, fundamentalmente de la cabeza con escasas posibilidades de obtenerlas completas, debido a su difícil desarticulación, estructura débil y morfología compleja.

La utilización de huesos aislados de la cabeza para uso docente en laboratorios de anatomía, presenta dificultades principalmente por su fragilidad y su uso se ve restringido por las bajas posibilidades de reposición. En ocasiones se utilizan piezas incluidas en acrílico, que si bien las protege y permite su visualización, no permiten al alumno el ejercicio de la totalidad de sus sentidos, dificultando los conceptos espaciales y tridimensionalidad para comprender mejor la estructura.

Comercialmente se venden cabezas óseas plásticas que pueden ser desarticuladas, que si bien puede ser un material representativo, en algunos casos adolecen de cualidades de textura, ausencia de forámenes, falta de cavidades y no menos importante tener la sensación que es un "hueso plástico". La morfología del cráneo es particularmente difí- cil de comprender, aunque actualmente para aprovechar la carencia de material, se utiliza la computación con el objeto de brindar al estudiante una herramienta para centrar su estudio y jerarquizarlo, aún sigue siendo imprescindible para su estudio examinar piezas anatómicas completas, desarticuladas o en cortes (D’Acuña \& Guiraldes, 1998).

Las dificultades actuales para obtener material cadavérico que esté disponible en forma permanente para el aprendizaje anatómico, han obligado a usar otros medios didácticos para suplir en su reemplazo, como una forma de evitar la destrucción del escaso material aún existente, ya que sigue siendo aconsejable la identificación de estructuras usando huesos, momificaciones, preparados en formol, imagenología (rayos X o resonancia magnética), modelos y es menos recomendable para dicho fin la utilización de láminas, dibujos o imágenes de programas computacionales (García-Hernández, 2003). Sin embargo es necesario que todo material didáctico represente adecuadamente la morfología, condición absolutamente necesaria para entender la disposición y fisiología de nuestro cuerpo.

\footnotetext{
* Facultad de Ciencias Naturales y Exactas. Universidad de Playa Ancha, Valparaíso-Chile.

**Facultad de Arte. Universidad de Playa Ancha. Valparaíso-Chile

Proyecto DIGI CNEI 08-0607. Universidad de Playa Ancha. Valparaíso-Chile
} 
La utilización conveniente de los materiales plásticos a lo largo de la historia de la anatomía ha sido un valioso apoyo a su enseñanza, ya sea como método de inclusión de piezas, sobre todo cuando estas deben permanecer en un medio líquido (Matamala et al., 1988; Oda \& Sant'Ana, 2003) como también en la inyección de resinas acrílicas autopolimerizables, las cuales han permitido elaborar moldes de los vasos sanguíneos seguida por la corrosión (Bustamante et al., 2007), lo que sumado a sus bajos costos, a incrementado su relevancia para la docencia e investigación. Referente a la utilización de resinas en la reproducción de preparaciones, su uso ha estado principalmente orientado a la toma de impresiones, al vaciado de algunas estructuras del sistema nervioso central, reproducción de macromodelos, coloración y protección de estructuras disecadas (Matamala et al.).

Las demandas didácticas de la anatomía humana han llevado a la necesidad de utilizar diferentes materiales para obtener moldes con diferentes fines, entre ellos podemos mencionar: la utilización del látex, yeso dental, caucho de silicona, arcilla, resinas de PVC y en el caso especifico de los huesos son varias las técnicas y materiales que deben combinar la estética con una adecuada adherencia a los márgenes óseos (Przybysz \& Scolin, 2008).

La técnica que a continuación se describe permite obtener réplicas óseas y está basada en materiales y procedimientos de restauración dental, técnicas artísticas y un detallado análisis morfoestructural, que enriquecen el procedimiento.

\section{MATERIAL Y MÉTODO}

Para la realización de la técnica se utilizaron los siguientes instrumentales e insumos: Gelatina de duplicado reversible (Castogel®), resinas de autopolimerización: Polimetacrilato de metilo (PMM) (polvo de acrílico) y Metilmetacrilato (MMA) (líquido) transparentes (Productos Dentales Marché $®$, Chile), fresadora con flexible y/o herramienta multiuso Dremel®, arenadora para prótesis dentales, balanza digital, pie de metro digital, calibrador, cera para troquelar, espátulas para cera, una cubeta de duplicado, tazas de goma, vasos precipitados, termómetro, mechero a gas, óleos de diferentes colores. Los huesos utilizados como modelo a replicar fueron: palatino, nasal, lagrimal y esfenoides.

Una buena reproducción debe partir de un buen modelo, por ello se tuvo especial cuidado en la selección y extracción de la pieza ósea. El análisis morfoestructural del hueso fue fundamental para los pasos siguientes, ya que fue necesario realizar restauraciones del modelo a reproducir, cuando este lo necesitó y se hizo necesario sellar parcialmente canales y forámenes con el fin de evitar retenciones del material de duplicación. Esta etapa de tratamiento y restauración se realizó con cera para troquelar.

Para hacer un duplicado completo del hueso, se hidrató previamente, con el fin de facilitar el desprendimiento del modelador una vez ocurrida la deshidratación. Como cubeta de duplicado utilizamos un recipiente plástico rectangular con tapa hermética, de acuerdo a las dimensiones y volumen del (os) hueso (s) a reproducir. Una vez hecho esto, trituramos el Castogel, cuya cantidad dependía del volumen de la cubeta de duplicado. Se calentó en un vaso precipitado a baño maría hasta que el gel pasó a un estado líquido, sin entrar en ebullición. Una vez que bajó su temperatura a $45^{\circ} \mathrm{C}$ se agregaron 2 a $3 \mathrm{ml}$ de agua corriente y se viertió el gel líquido en la cubeta de duplicado formando una base de $1 \mathrm{~cm}$ por lado.

Una vez realizado este paso, se puso el hueso en el gel de manera expulsiva para facilitar su desmolde. Se virtió nuevamente gel, hasta cubrir la mitad del preparado, dejando gelificar por al menos 30 min a temperatura ambiente. Posteriormente se humedeció la superficie superior con agua corriente y se vertió el resto del gel hasta cubrir completamente la pieza sobre $1 \mathrm{~cm}$ de su región más prominente y nuevamente se dejó gelificar por el mismo lapso de tiempo. Una vez terminada esta etapa, se separaron las dos mitades del molde de gel y se extrajo la pieza original.

La segunda etapa del proceso, consistió en preparar el color, destacando que las reproducciones no son pintadas, sino que el color base y las pátinas fueron incluidas en el acrílico. El color base utilizado correspondió a un tono similar a los huesos obtenidos de cementerio, con sus pátinas características, sin realizar un blanqueado. Sin embargo el color depende de las necesidades del usuario de la técnica. El óleo mezclado se combinó con el monómero dejando el líquido coloreado.

El tercer paso es la preparación del acrílico. La cantidad de acrílico a preparar depende del modelo a reproducir. La proporción de acrílico que utilizamos fue de 2:1 (polvo: líquido). Para las pátinas y manchas se agregó una pequeña pincelada de óleo "tierra de siena natural" cuando el acrílico se encuentra en estado arenoso previo a moldear. Una vez realizada la mezcla se virtió en estado arenoso en los moldes de gel por ambas caras, se juntaron y se dejó fraguar por al menos $30 \mathrm{~min}$. El paso siguiente fue el desmolde, donde se extrajo la pieza y se procedió a recortar los excesos, utilizando una fresadora con flexible con una punta fina y/o la herramienta multiuso Dremel ${ }^{\circledR}$ y finalmente para eliminar los residuos se utilizó la arenadora. 


\section{RESULTADOS}

Las piezas obtenidas tuvieron un alto grado de fidelidad y los duplicados requirieron de poca intervención, en donde básicamente se eliminaron los residuos de acrílico sobrante.

$\mathrm{Al}$ analizar cada preparado anatómico en forma particular, se pueden destacar algunos detalles morfológicos que demuestran la alta precisión de los duplicados, entre ellos podemos mencionar: En la réplica de hueso palatino podemos observar en su vista medial (Fig.1 A) su lámina horizontal con su borde medial grueso y serrado (1) para articular con el del otro lado en la línea mediana. Se evidencia claramente la cresta conchal (2) y la cresta etmoidal (3). Además podemos apreciar claramente en su cara lateral (Fig.

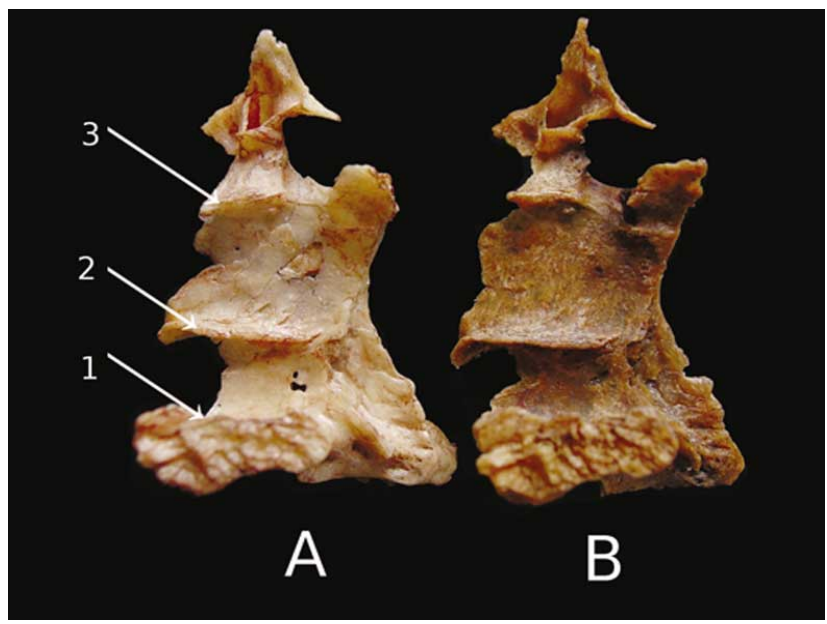

Fig. 1. Hueso palatino .Vista medial. (A) Réplica final. 1. Lámina Horizontal.; 2. Cresta Conchal; 3.Cresta Etmoidal (B) Pieza original.

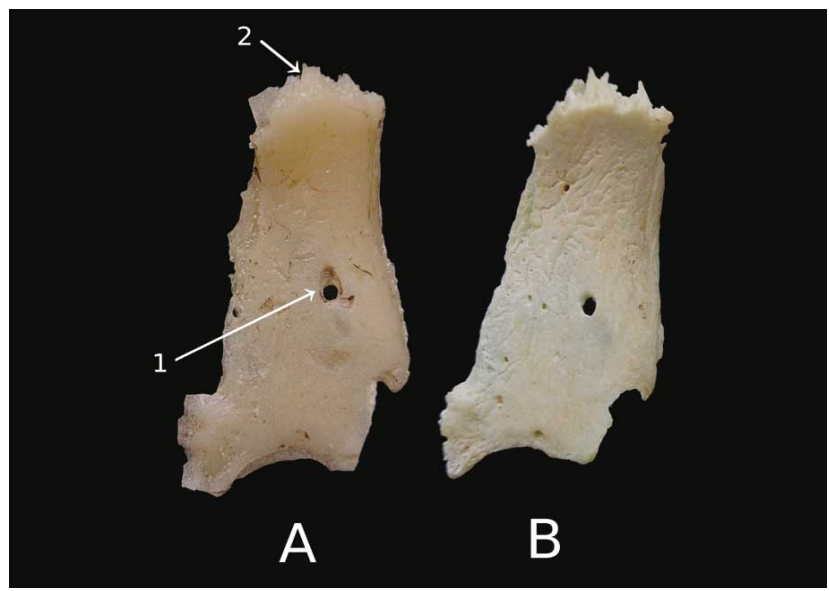

Fig. 3. Hueso nasal. Vista anterior. A) Réplica final. 1. Foramen vascular; 2. Margen superior (B) Pieza original.
2 A) las rugosidades del proceso piramidal para articular con el maxilar (1), el surco palatino mayor (2) y el proceso orbitario (3). En el hueso nasal (Fig. 3 A) podemos destacar algunas formaciones anatómicas como la presencia de un foramen vascular en su cara anterior (1) y su margen superior dentado (2). En la réplica de hueso lagrimal (Fig. 4 A) se puede observar la cresta lagrimal posterior (1), el surco lagrimal (2) y la fosa del saco lagrimal (3).

La réplica de hueso esfenoides permite apreciar en mejor medida los excelentes resultados de la técnica. En una vista superior (Fig. 5) se observa la língula esfenoidal (1), la presencia de los forámenes redondo y oval (2 y 4) y detalles como la textura y el color del margen frontal. Por otra parte, en la vista posterior (Fig. 7) se puede ver la detallada precisión del margen escamoso (1), la cara cerebral del ala mayor (2) y el proceso clinoides anterior (3). Final-

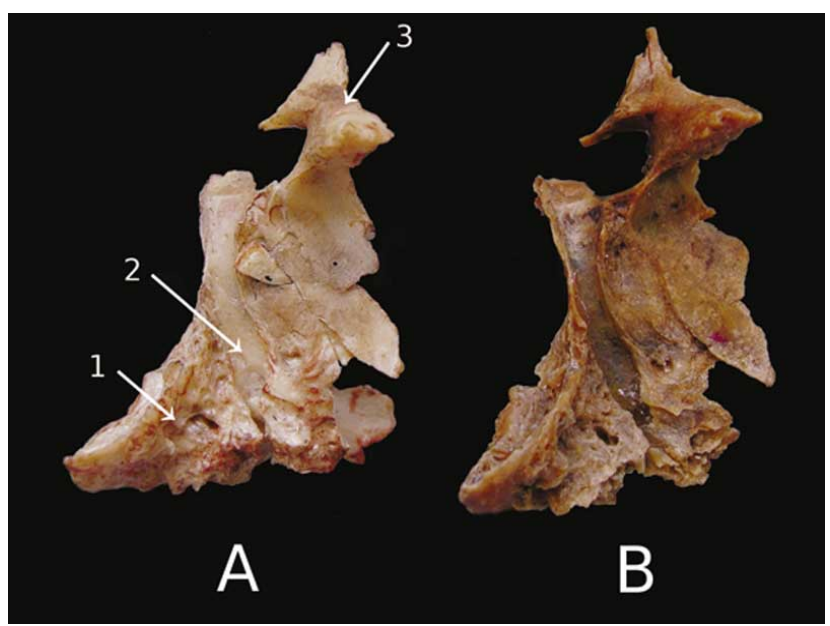

Fig. 2. Hueso palatino. Vista lateral. (A) Réplica final. 1. Proceso piramidal; 2.Surco palatino mayor; 3. Proceso Orbitario. (B) Pieza original.

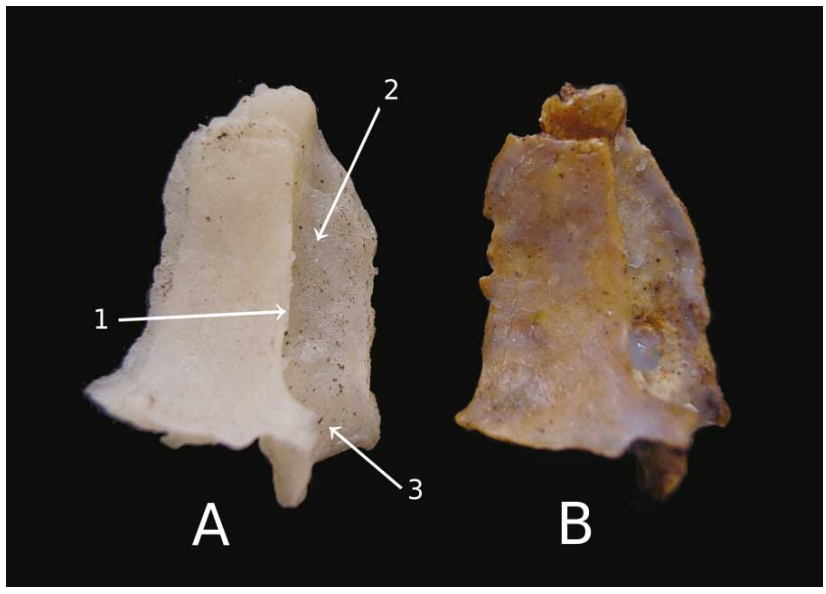

Fig. 4. Hueso lagrimal. Vista lateral. A) Réplica final. 1. Cresta Lagrimal posterior; 2. Surco Lagrimal; 3. Fosa del Saco Lagrimal. (B) Pieza original restaurada. 


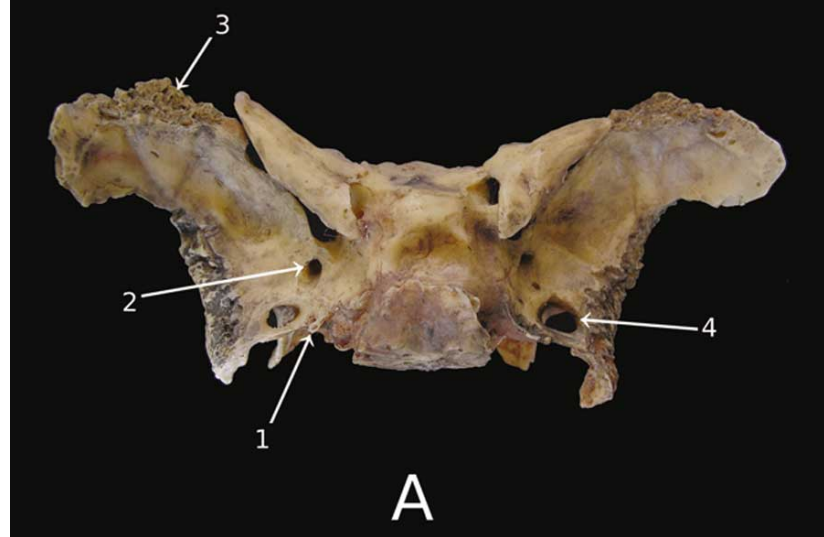

Fig. 5. Hueso esfenoides. Vista superior. Réplica final. 1. Língula esfenoidal. ;2.Foramen redondo; 3. Margen frontal; 4. Foramen oval.

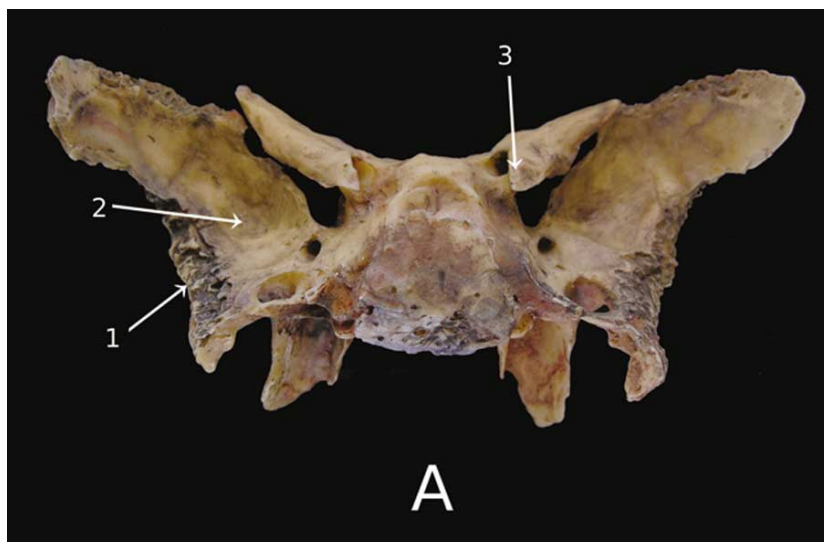

Fig. 7. Hueso esfenoides. Vista posterior. Réplica final. 1 Margen escamoso; 2. Cara cerebral del ala mayor; 3. Proceso clinoides anterior.

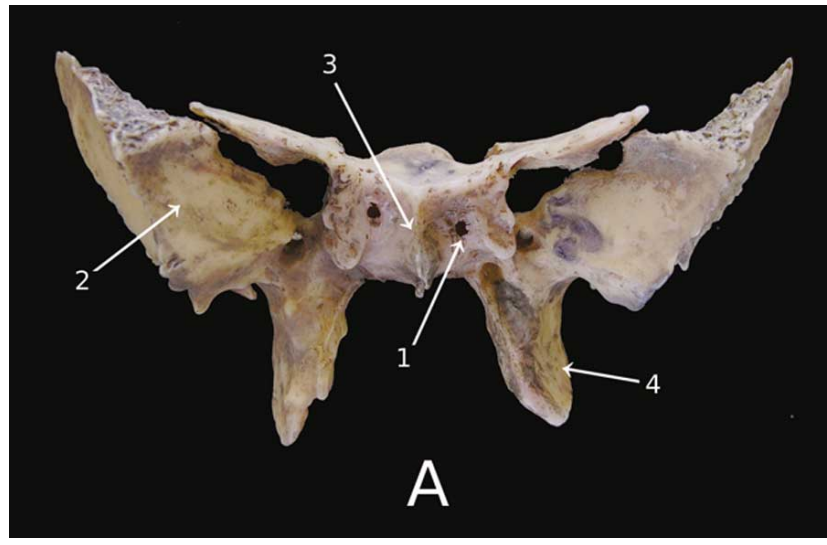

Fig. 9. Hueso esfenoides. Vista Anterior .Réplica final. 1. Cuerpo del esfenoides y apertura del seno esfenoidal; 2. Cara orbitaria del ala mayor; 3. Cresta esfenoidal; 4. Lámina lateral del proceso pterigoides.

mente en una vista anterior de la misma pieza (Fig. 9) se puede apreciar entre otros, su cuerpo en el cual se conservo las aperturas del seno esfenoidal (1) y la cresta esfenoidal (3). También se puede evidenciar la cara orbitaria lisa (2) y el proceso pterigoides con sus dos láminas (4).

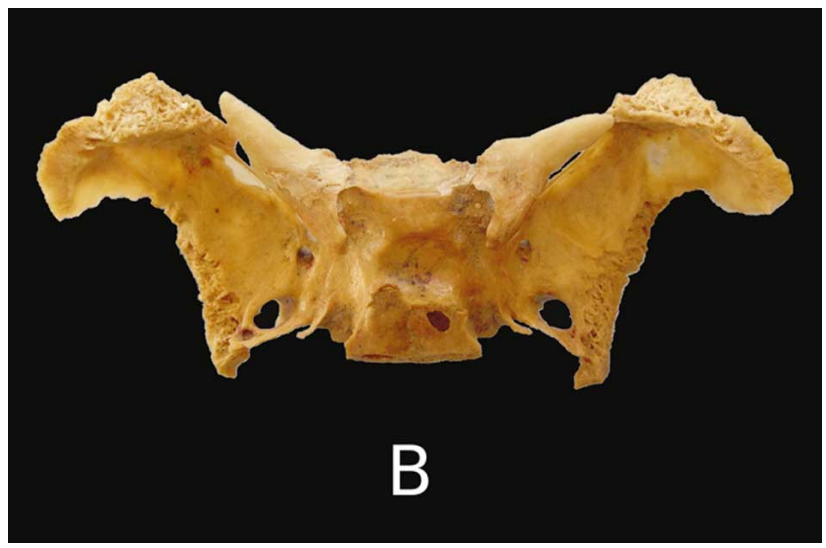

Fig. 6. Hueso esfenoides. Vista superior. Pieza original

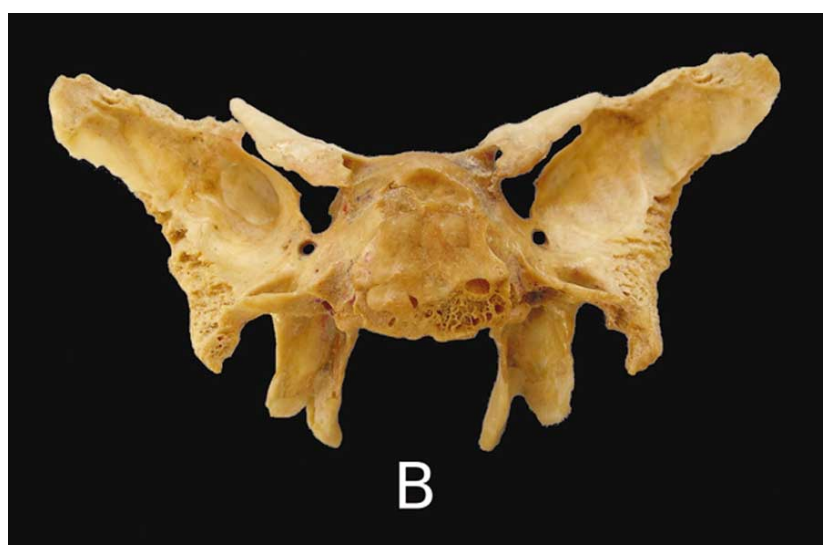

Fig. 8. Hueso esfenoides. Vista posterior. Pieza original.

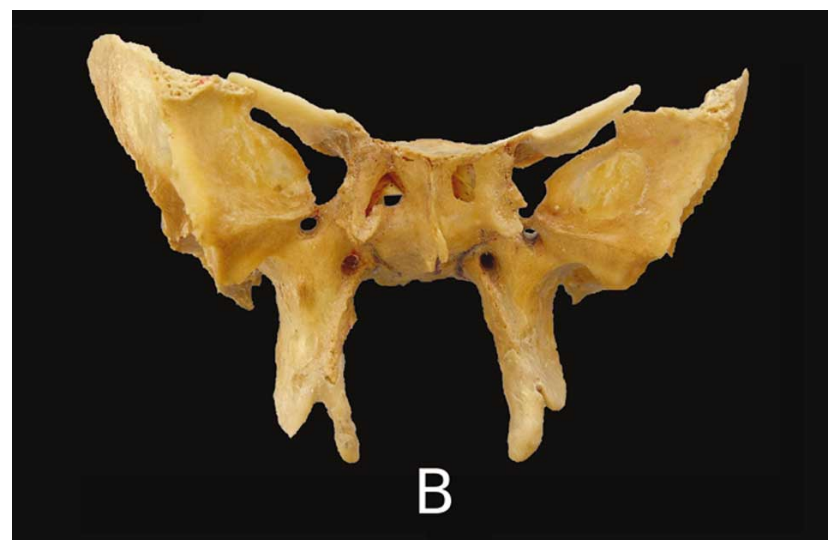

Fig. 10. Hueso esfenoides. Vista anterior. Pieza original.

Es importante destacar que cada réplica conserva los parámetros morfológicos de su original, como rugosidades, surcos, forámenes, procesos, entre otros. La masa de las replicas estuvo determinada por la mayor o menor presencia de hueso compacto y/o esponjoso en la pieza original. En el caso de los duplicados de acrílico, cuyo patrón original tenía mayor presencia de hueso compacto, la masa de la réplica v/s el hueso original fue equilibrada una vez que se evaporó el 100\%del monómero. 


\section{DISCUSIÓN}

Con respecto a la utilidad de la reproducciones óseas, coincidimos con Segovia \& Moreno (1989) que señala que el uso de modelos tamaño natural y que representen fielmente la pieza anatómica es un recurso comprobadamente efectivo en el proceso de enseñanza-aprendizaje de la Anatomía Humana y creemos que la duplicación de huesos con rigurosidad morfoestructural y su posterior utilización, es una contribución con un medio alternativo muy similar a la estructura normal y que puede ser una excelente herramienta para enseñanza y/o investigación.

Es importante destacar la potencialidad de las reproducciones óseas de calidad en diferentes parámetros morfológicos usando acrílicos, ya que pueden ser usados como simuladores quirúrgicos de entrenamiento (Okada et al., 2010)

En nuestro laboratorio ya habíamos utilizado esta técnica para reproducir huesos de miembros u otros de esqueleto axial y esta investigación nos permitió aplicar la técnica para reproducir preparados óseos de difícil obtención como lo son algunos huesos de la cabeza, que presentan diferentes retos, dependiendo de la pieza a reproducir como por ejemplo la presencia de senos, sus dimensiones y zonas extremadamente delgadas.

Esta técnica permite tener un material tridimensional con las mismas características del original que no requiere mantención, siendo una ventaja del uso de este tipo de resina acrílica su fácil preparación, y su costo asequible (Kurzer, 2006)

Se debe destacar que dentro de esta técnica se requiere un análisis morfoestructural de las piezas en todas las etapas del proceso, comenzando en la extracción del hueso, pasando por su restauración, la obtención del molde de duplicación y las futuras replicas, lo cual enriquece la técnica y la calidad de la morfoestructura de las reproducciones.

A diferencia de otras técnicas para reproducir piezas óseas (Przybysz \& Scolin) pensamos que el gel duplicador utilizado copia fielmente el modelo, preservando todas sus características, forámenes, rugosidades, salientes y depresiones, lo cual es ideal para obtener modelos a partir de huesos de la cabeza ósea como los obtenidos en esta investigación.

VILLARROEL, G. M. \& MEDINA, O. E. Anatomical technique to restore and/or reproduce human bone pieces difficult toobtain for research and teaching science. Int. J. Morphol., 29(2):532-536, 2011.

SUMMARY: A technique to reproduce and/or restore human bones for teaching, scientific, academic or artistic purposes is described. This technique allows us to obtain optimal preparations in texture, color, allowing three-dimensionality as well as subtle morphological details of bone pieces. The technique is applied for reproducing human bone pieces that are difficult to obtain, as are the bones of the head. However all types of bone pieces can be used. Our results confirm that this technique has a high degree of reliability compared with the original piece. Therefore the technique can be very useful in teaching and research.

KEY WORDS: Anatomic techniques; Acrylics; Bones reproduce; Didactic materials; Artistic anatomy.

\section{REFERENCIAS BIBLIOGRÁFICAS}

Bustamante, M.; Prieto, R. \& Binvignat, O. Preservación de Placenta Humana: Técnica Anatómica. Int. J. Morphol., 25(3):545-8, 2007.

D`acuña, E \& Guiraldes del C., H. Anatomía del cráneo en páginas Web. Rev. Chil. Anat., 16(1):115-66,1998.

García-Hernández, F. Evaluación del aprendizaje práctico de la anatomía humana para odontología en la universidad de Antofagasta, Chile. Int. J. Morphol., 21(1):43-7, 2003.

Kurzer, M. Estudio comparativo de dureza en dientes artificiales fabricados con diferentes tipos de resinas acrílicas. Revista EIA, 6:121-8, 2006.

Matamala, F.; Olave, E.; Henríquez, J. \& Chavez, R. Experiencia de siete años en el Diseño y uso de material didáctico anatómico de bajo costo. An. Anat. Norm., 6(6):208-13, 1988.

Oda, J. \& Sant'Ana, D. Técnica de inclusão em resina de cortes transversais da medula espinhal coradas pelo método de mulligan. Int. J. Morphol., 21(1):49-92, 2003.

Okada, D; de Sousa, A; Huertas, R. \& Suzuki, F. Surgical simulator for temporal bone dissection training. Braz. J. Otorhinolaryngol., 76(5):575-8, 2010.

Przybysz, C. \& Scolin, E. Técnica anatômica: confecção de modelos em resina a partir de vértebras humana. F@PCiencia, 2(10), 2008. Disponible en: http://www.fap.com.br/fapciencia/ 002/edicao_2008/010.pdf. Acceso el 23/10/2010

Segovia, J. \& Moreno, E. Uso de yeso emparafinado en la fabricación de modelos anatómicos. An. Anat. Norm., 7:158-9, 1989.

Dirección de correspondencia:

Prof. Mauricio Villarroel Guerra

Departamento disciplinario de Biología

Facultad de Ciencias Naturales y Exactas

Universidad de Playa Ancha

Casilla 34-V

Valparaíso-CHILE

Email: mvillarr@upla.cl.

Recibido : 28-12-2010

Aceptado: 16-03-2011 